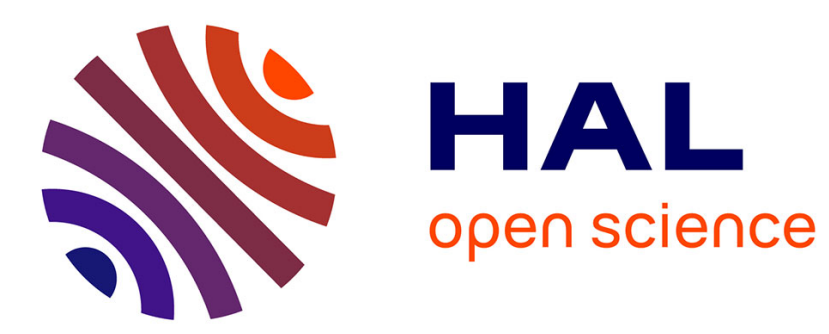

\title{
Snowballs, quantum solvation and coordination: lead ions inside small helium droplets
}

\author{
P. Slavícek, Marius Lewerenz
}

\section{To cite this version:}

P. Slavícek, Marius Lewerenz. Snowballs, quantum solvation and coordination: lead ions inside small helium droplets. Physical Chemistry Chemical Physics, 2010, 12, pp.1152-1161. 10.1039/b918186e . hal-00830349

\section{HAL Id: hal-00830349 \\ https://hal.science/hal-00830349}

Submitted on 4 Jun 2013

HAL is a multi-disciplinary open access archive for the deposit and dissemination of scientific research documents, whether they are published or not. The documents may come from teaching and research institutions in France or abroad, or from public or private research centers.
L'archive ouverte pluridisciplinaire HAL, est destinée au dépôt et à la diffusion de documents scientifiques de niveau recherche, publiés ou non, émanant des établissements d'enseignement et de recherche français ou étrangers, des laboratoires publics ou privés. 


\title{
Snowballs, quantum solvation and coordination: lead ions inside small helium droplets
}

\author{
Petr Slavíček $^{a}$ and Marius Lewerenz ${ }^{* b}$
}

\author{
Received 3rd September 2009, Accepted 12th November 2009 \\ First published as an Advance Article on the web 10th December 2009 \\ DOI: $10.1039 / b 918186 e$
}

\begin{abstract}
$A b$ initio calculations are used to construct an analytical many-body potential for $\mathrm{Pb}^{2+} \mathrm{He}_{n}$ and $\mathrm{Pb}^{+} \mathrm{He}_{n}$ clusters which accounts for non pairwise additive interactions. The potential surface reproduces the global minima for cluster sizes ranging from $n=1$ to $n=16$ obtained from explicit $a b$ initio calculations and found in a previous search for ultrahigh coordination numbers. Ground state energies and structures obtained by accurate diffusion quantum Monte Carlo calculations are used to investigate if quantum effects qualitatively affect the formation of coordination shells. For $\mathrm{Pb}^{2+}$ doped clusters a first solvation shell is closed at $n=12$ and gradually softened by additional helium atoms which start to form a distinct second shell only at $n=16$. Spin-orbit coupling profoundly influences the structure of $\mathrm{Pb}^{+} \mathrm{He}_{n}$ clusters and causes a gradual structural evolution without pronounced solvation shells.
\end{abstract}

\section{Introduction}

The relation between neighboring spherical objects is described with a wide variety of concepts depending on the scientific field. The mathematical problem of packing of hard spheres gives rise to the definition of the kissing or Newton number. ${ }^{1}$ Related terms are contact, parking or caging numbers. In chemistry the number of interacting neighbors is usually described by the coordination number. In coordination chemistry this number is always found to be between 1 and $12,{ }^{2}$ the extreme values being rather rare.

The search for the highest possible coordination numbers in chemically bound systems has recently inspired Hermann et al. to perform electronic structure calculations on the $\mathrm{Pb}^{2+} \mathrm{He}_{n}$ systems $^{3}$ in extension of related earlier work on yttrium complexes. ${ }^{4,5}$ This study identified $\left(\mathrm{PbHe}_{15}\right)^{2+}$ as a stable structure in which all 15 helium atoms form a single shell around the central ion. Helium is clearly an unusual ligand of extremely low reactivity and additionally exhibits significant quantum effects such that ground-state-averaged structures can qualitatively deviate from electronic equilibrium structures. Precisely because of these quantum effects liquid helium droplets have, however, recently attracted considerable attention as a weakly perturbing solvent for spectroscopy and reaction dynamics. ${ }^{6-10}$ The possibility of strong bonds with cations and the existence of thermodynamically stable helium compounds has already been discussed more than 20 years ago ${ }^{11}$ and the available information on small ionic helium complexes has been reviewed recently. ${ }^{12}$

The common case of hydrated metal ions in aqueous solutions leads to the natural question of the distinction between coordination and solvation. This distinction is usually

${ }^{a}$ Department of Physical Chemistry, Institute of Chemical Technology Prague, Technická 6, 16628 Prague 6, Czech Republic

${ }^{b}$ MSME, FRE 3160 CNRS, Bât. Lavoisier, Université Paris-Est

(Marne la Vallée), 77454 Marne la Vallée Cedex 2, France.

E-mail: Marius.Lewerenz@univ-paris-est.fr based on interaction strength and nearest neighbor distances. The interaction should be strong enough to prevent exchange such that the coordination complex is a clearly identifiable object. The presence of quantum exchange in a solvent like helium makes the distinction between coordinated atoms and solvent atoms obviously very difficult. It has been known for a long time ${ }^{13}$ from ion mobility studies in bulk liquid helium that ions carry along a coating of strongly localized helium atoms which is often referred to as a "snowball". This object can be viewed as a coordination complex solvated in a solvent composed of the same atoms. The snowball might, however, well be a dynamical object with a well defined average mass and number of nearest neighbor helium atoms but involved in active particle exchange with its environment.

The goal of this article is to shed some light on the structure of highly coordinated systems in the presence of pronounced quantum effects. Lead ions interacting with helium are ideal test systems because high coordination numbers have been predicted in electronic structure calculations which do not take into account vibrational quantum effects. ${ }^{3}$ Experimental evidence for the relative stabilities of mixed lead/helium ionic complexes comes from recent ionization experiments on lead atoms implanted into large neutral helium clusters. ${ }^{10,14}$ These observations indicate the existence of particularly stable ("magic") $\left(\mathrm{PbHe}_{12}\right)^{2+}$ and $\left(\mathrm{PbHe}_{17}\right)^{+}$ions and do not hint at any special stability for $\left(\mathrm{PbHe}_{15}\right)^{2+}$. Very recent mass spectrometric experiments provide valuable additional information on the stability and possible structure of a variety of other positively or negatively charged ionic helium complexes. ${ }^{15}$

The overwhelming zero point energy in neutral pure and doped helium clusters leads to extreme delocalization of the helium atoms such that conventional notions of geometrical structures or solvation shells become largely invalid. The stronger interactions in charged clusters are known to lead to a higher degree of particle localization ${ }^{16-19}$ but the structures resulting from quantum averaging over the nuclear motion ground state wave function can be significantly 
different from 'classical' structures. ${ }^{20-22}$ Under these circumstances the existence of a high coordination global minimum structure for $\left(\mathrm{PbHe}_{15}\right)^{2+}$ on the electronic ground state surface does not guarantee the existence of such a structure in the true quantum ground state including all degrees of freedom. In order to verify if the structures identified by Hermann et al. ${ }^{3}$ are robust with respect to zero point effects we undertook the construction of a global analytical potential surface for $\left(\mathrm{PbHe}_{n}\right)^{2+}$ beyond the pairwise additive approximation and based on advanced $a b$ initio calculations which reproduces the known minimum energy structures.

The observation of ion abundances which are indicative of shell closure effects for $\mathrm{Pb}^{+}$in helium ${ }^{14}$ motivated us to extend our study to this system which poses additional challenges due to its open shell electronic structure and the presence of strong spin-orbit coupling. Spin-orbit coupling is expected to have a significant effect on the cluster structures due to the strong mixing between the deep zero order ${ }^{2} \Pi$ interaction and the very shallow ${ }^{2} \Sigma^{+}$potential of $\mathrm{Pb}^{+} \mathrm{He}$ which markedly reduces the anisotropy of the interaction potential.

After a description of our electronic structure approach and a summary of some relevant details of our diffusion Monte Carlo (DMC) methodology we discuss the construction of our many body potential models. Optimum and quantum averaged structures and the corresponding energies are then discussed for the two distinct cases of strong coordination $\left(\mathrm{Pb}^{2+} \mathrm{He}_{n}\right)$ and weak coordination $\left(\mathrm{Pb}^{+} \mathrm{He}_{n}\right)$. We conclude with a brief analysis of the concept of solvation shells in a quantum context.

\section{Computational details}

\section{A Electronic structure calculations}

The pair interaction potential between the $\mathrm{Pb}^{2+}$ ion and helium atoms has been calculated at the $\operatorname{CCSD}(\mathrm{T})$ level using a supramolecular approach. The standard counterpoise correction of the basis set superposition error was applied. ${ }^{23}$ The interaction energy converges relatively slowly with the basis set size due to the important contribution of the dispersion interaction to the binding. We have therefore applied rather large aug-cc-pV5Z basis sets both for lead and helium. ${ }^{24,25}$ The small-core relativistic effective core potential of the Stuttgart group ${ }^{24}$ was used to describe the 60 inner electrons of the lead atom. To accelerate the convergence of the binding energy we have also placed a small set of bond functions in the midbond region. In particular, three $\mathrm{s}$ and three $\mathrm{p}$ functions with exponents of $0.9,0.3$ and 0.1 were added.

The many-body contribution to the intermolecular potential converges much faster with the basis set size due to its dominantly electrostatic character. The three-body interaction is essentially converged at the CCSD(T)/aug-cc-pVTZ level, which has therefore been used to construct this contribution to the total interaction. These $\operatorname{CCSD}(\mathrm{T})$ based two- and three body terms were used to define our analytical many body model. Structural predictions for larger clusters based on this model (see section III A) were checked by direct electronic structure calculations at the DFT level. These calculations used the PBE0 functional ${ }^{26}$ in combination with much smaller basis sets of triple zeta and double zeta quality for lead and helium. Despite the known deficiencies of DFT for the description of dispersion interactions this combination leads to a reasonable description of the energetics of both $\mathrm{Pb}^{2+} \ldots \mathrm{He}$ and $\mathrm{He} \cdot \mathrm{He}$ dimers while it still allows calculations on larger clusters. Note that the purpose of the DFT calculation is only to check if our many body models exhibit any major deficiencies.

The nature of the interaction was analyzed using symmetryadapted perturbation theory (SAPT) $)^{27}$ as implemented in MOLPRO. $^{28}$ SAPT was specifically used to extract the induction terms from the overall interaction energy.

The interaction between ground state $\mathrm{Pb}^{+}$ions and helium is more complicated since this ion has an unpaired electron occupying a p orbital. The three lowest asymptotically degenerate states $\left({ }^{2} \Sigma^{+}\right.$and a degenerate pair of ${ }^{2} \Pi$ states) separate at finite distances. Pair interaction potentials for all three states were calculated using the unrestricted $\operatorname{CCSD}(\mathrm{T})$ method with a aug-cc-pV5Z basis set, using the above mentioned set of midbond functions. Note that the $\mathrm{UCCSD}(\mathrm{T})$ method is based on the RHF wavefunction (RHF-UCCSD method). The spin contamination of the resulting wavefunction was small for all the calculations. The symmetry of the wavefunctions was used in the calculations. The interaction between the $\mathrm{Pb}^{+}$ion and helium is strongly affected by spin-orbit interaction which leads to almost complete mixing of the ${ }^{2} \Sigma^{+}$state and the $\Omega=\frac{1}{2}$ component of the ${ }^{2} \Pi$ state. As a check for our many body model we have also optimized structures of larger clusters, using again the DFT method with the PBE0 functional. To show the structure-forming role of the spin-orbit effect, we have performed these optimizations both with and without inclusion of the spin-orbit interaction.

The $\operatorname{CCSD}(\mathrm{T})$ and UCCSD(T) electronic structure calculations have been carried out with the Molpro suite of programs $^{28}$ and the DFT optimizations with and without spin-orbit interaction were performed with the NWCHEM code. $^{29}$

\section{B Diffusion Monte Carlo calculations}

The DMC method exploits an isomorphism between the time dependent Schrödinger equation and a multi dimensional diffusion equation and a random walk technique to solve the diffusion problem which corresponds to a given quantum problem. The general principles and important technical details of the method are discussed in several classical publications. $^{30-33}$ The statistical accuracy of the method can be significantly enhanced by the use of a trial wave function $\Psi_{\mathrm{T}}$ which resembles the exact but unknown eigenfunction $\Psi$. In the unrealistic but formally interesting limit $\Psi_{\mathrm{T}}=\Psi$ all statistical errors vanish. Our specific implementation uses continuous weights and fixed random walker ensemble sizes with stochastic population control as described in more detail in earlier publications. ${ }^{22,34,35} \mathrm{We}$ will restrict the present discussion to some special aspects which are of relevance for the current application which tries to resolve structural questions related to expectation values. 
DMC is essentially a projection method which projects an eigenfunction $\Psi$, usually the ground state wave function unless special constraints are applied, from an initial wave function by propagation in imaginary time $\tau$. Formally the exact solution is reached as $\tau \rightarrow \infty$. While DMC is an exact method in the sense that all errors vanish if certain technical parameters tend to limiting values, any actual calculation requires careful monitoring of statistical and various systematic errors in calculated energies and even more so for expectation values.

Aside from the problem of defining the asymptotic regime $\tau \approx \infty$ where $\Psi(\tau) \approx \Psi$ and an arbitrary amount of information can be collected by simply continuing the projection process (the main part on any DMC calculation) errors arise from two aspects of the projection process. In the absence of knowledge of the exact projector (Green's function)

$$
\Psi(x, \tau)=\int G\left(x, x^{\prime}, \tau\right) \Psi\left(x^{\prime}, 0\right) \mathrm{d} x^{\prime}
$$

the projection time is divided into steps $\Delta \tau$ over which a short time approximation of the projector can be applied.

$$
\Psi(x, \tau+\Delta \tau)=\int G\left(x, x^{\prime}, \Delta \tau\right) \Psi\left(x^{\prime}, \tau\right) \mathrm{d} x^{\prime}
$$

This practical necessity leads to a time step error which is discussed in the published literature ${ }^{32}$ and imposes either the use of sufficiently small time steps to make this error smaller than the statistical sampling errors of the method or an extrapolation of results obtained at several values of $\Delta \tau$ to the limit $\Delta \tau=0$.

A second error which is much less well documented in the published literature but which turns out to be often more important is related to the actual projection process. Within DMC the product $\Psi \Psi_{\mathrm{T}}$ is represented by an ensemble of random walkers of size $N$. The computation of the projection integral in eqn (2) is replaced by sampling from $G\left(x, x^{\prime}, \Delta \tau\right)$. This procedure is exact if configuration space is covered by an infinite number of random walkers. For any finite size $N$ of the random walker ensemble the result of the projection over a step $\Delta \tau$ deviates from the exact result $\Psi_{\infty}$ by a random fluctuation $\delta$ with zero mean.

$$
\begin{aligned}
\Psi_{N}(x, \tau) & =\Psi_{\infty}(x, \tau)+\delta(x, \tau) \\
\langle\delta(x, \tau)\rangle_{\tau} & =0
\end{aligned}
$$

Even if the projection process had resulted in the exact wave function at some sufficiently large $\tau$ this situation would be immediately destroyed by fluctuations! It is clear that the amplitude of $\delta$ tends to zero as $N \rightarrow \infty$. For any finite $N$ the deviations $\delta$ can be formally expanded into the full set of eigenfunctions of the system. A simple analysis shows that the energy expectation value $E_{N}$ of this mixed wave function is systematically above the exact value and that we have

$$
E_{N}=E_{\infty}+\frac{c}{N}
$$

with an unknown coefficient $c$ which depends on the noisiness of the simulation and thereby mainly on the quality of the trial wave function.

An efficient trial wave function is a very good approximation to the exact ground state wave function and its construction requires detailed structural information. In the present application we want to generate (potentially unexpected) structural information and we have to use functions which provide very little structural information in order to avoid a systematic bias. The only reliable information which can be safely exploited for the construction of $\Psi_{\mathrm{T}}$ concerns the limiting behavior as two particle get very close to each other or as a particle tries to leave the cluster. The use of simple DMC without any trial wave function, equivalent to posing $\Psi_{\mathrm{T}}=1$, is not only fundamentally incorrect (any trial wave function has to satisfy all usual conditions for a quantum mechanical wave function) but leads to large statistical noise. Our trial wave functions of deliberately modest quality place us relatively close to this limit while being formally correct. In return we had to use ensemble sizes of up to 16000 random walkers to make the energy bias from eqn (5) smaller than the statistical errors and very long accumulation times for converged descendent weights (see below).

The introduction of a trial wave function into the DMC method leads to a convection term in the diffusion equation which is solved in our implementation by the classical low order integration scheme of ref. 31 resulting in a time step error of the form

$$
E(\Delta \tau)=E_{0}+c_{1} \Delta \tau+\mathcal{O}\left(\Delta \tau^{2}\right)
$$

This scheme allows us to impose detailed balance on the random walks by a Metropolis scheme such that the number density of random walkers is guaranteed to be proportional to $\Psi_{\mathrm{T}}^{2}$ at any $\Delta \tau .^{31,32}$ Formally more accurate higher order propagation schemes with leading time step errors $\Delta E \propto \Delta \tau^{k}$, $k>1$ have been proposed by several authors ${ }^{36,37}$ but the complexity of these algorithms prevents the enforcement of detailed balance. The latter turns out to dramatically reduce the absolute size of the time step error (the coefficient $c_{1}$ in eqn (6)) such that the advantage of these higher order schemes remains unclear in practice even for energy computations. Imposing a number density $\propto \Psi_{\mathrm{T}}^{2}$ is, however, essential for the identification of the weights of the random walkers as an unbiased estimator of the ratio $\Psi / \Psi_{\mathrm{T}}$ which is a key requirement of all exact algorithms for $\Psi^{2}$ sampling. Unbiased expectation values are essential for the structural features which we want to study here. All expectation values for scalar properties and distribution functions reported in this article have been computed by the descendent weighting procedure. ${ }^{38,39}$

In the presence of both time step and ensemble size errors all energies reported here were obtained by double extrapolations towards zero time step and infinite ensemble size using the known dependencies $E(N, \Delta \tau)$. The DMC calculations were carried out with random walker ensemble sizes between 500 and 16000 and time steps ranging from $100 \mathrm{E}_{\mathrm{h}}^{-1}$ to $10 \mathrm{E}_{\mathrm{h}}{ }^{-1}$. After relaxation of initial distributions averages were typically collected over 500 blocks of steps of length $5000 \mathrm{E}_{\mathrm{h}}{ }^{-1}$. Each block of steps produces one value for the growth energy $E_{\text {grow }}$ and a local energy block average. These block averages and the growth energy samples were analysed for serial correlation, giving correlation lengths $l_{\text {corr }}$ between 1.3 and 2.5. The $1 \sigma$ error bars for individual results were corrected for this residual serial correlation with the standard formula $\sigma_{\text {true }} \approx \sigma_{\text {raw }} \sqrt{l_{\text {corr }}}$ and used as weights in the extrapolation to infinite ensemble 
size and zero time step. Statistical errors for extrapolated energies are computed by standard techniques of weighted least squares fitting. ${ }^{40}$

\section{Results and discussion}

\section{A Construction of the many body surfaces}

$1 \mathbf{P b}^{2+}-$ He. The nature of the interaction between the $\mathrm{Pb}^{2+}$ ion and neutral helium atoms is rather complicated and needs to be modelled beyond a pair potential approximation. The attractive part of the interaction is dominated by the interaction between the charge of $\mathrm{Pb}^{2+}$ and the induced dipole on the helium atoms and can be written in atomic units as

$$
V_{\mathrm{CID}}(r)=-\frac{q_{\mathrm{Pb}}^{2} \alpha_{\mathrm{He}}}{2 r^{4}}
$$

More effects, however, need to be taken into account. First, $\mathrm{Pb}^{2+}$ is an ion with an extended charge distribution. As a consequence, the induced dipole moment on the helium atom is larger than the value predicted from standard electrostatic formulas. The size of $\mathrm{Pb}^{2+}$ also leads to a more pronounced electrostatic interaction between the ion and the helium atom electron clouds than one would anticipate from the model of point charge and point dipole. Finally, the dipoles induced on the helium atoms induce additional dipole and quadrupole moments on the $\mathrm{Pb}^{2+}$ ion, contributing again to the bonding.

In the absence of detailed knowledge of the diverse contributions to the interaction we decided to fit the two-body interaction by an analytical model and to model the dominant induction contributions. In our model, the potential energy surface for $\left(\mathrm{PbHe}_{n}\right)^{2+}$ clusters is constructed as follows:

$$
V=\sum_{i=1}^{n_{\mathrm{He}}}\left(V_{\text {pair }}^{i}+V_{\text {ind }}^{i}-V_{\text {ind }}^{i, \text { dimer }}\right)+\sum_{i=1}^{n^{n} \mathrm{He}} \sum_{j=i+1}^{{ }^{n} \mathrm{He}}\left(V_{\text {pair }}^{i j}+V_{\text {ind }}^{i j}\right)
$$

where $V_{\text {pair }}^{i}$ is the ab initio pair interaction between the $\mathrm{Pb}^{2+}$ ion and the $i$-th helium atom and $V_{\text {pair }}^{i j}$ denotes the pair interaction between helium atoms. The pairwise terms are then augmented by the non-additive interactions between induced moments: $V_{\text {ind }}^{i}$ is the interaction energy between the induced moments on lead and induced moments on helium atoms and is in fact a many-body term since it arises from the response of the lead ion to the complete set of helium atoms, $V_{\text {ind }}^{i j}$ describes interactions between induced moments on helium atoms, $V_{\text {ind }}^{i, \text { dimer }}$ is the interaction between induced electric moments on lead and helium for a dimer and has to be subtracted since this term is already implicitly included in the pair contribution explicitly calculated by electronic structure methods. The interaction between induced electric moments is responsible for the non-additivity of the interaction.

For the $V_{\text {pair }}^{i j}$ part of the helium-helium pair interaction, we used the HFD-B potential model of Aziz ${ }^{41}$ and the very recent and highly accurate pair potential of Jeziorska et al. ${ }^{42}$ The large number of evaluations of the potential surface is the highest cost factor in a DMC calculation. The vast majority of the production calculations were carried out with the older Aziz potential due to its much higher computational speed.
Calculations with the roughly three times more expensive potential from ref. 42 were performed for several cluster sizes and the energy difference with respect to the HFD model was additionally computed with our correlated sampling technique. ${ }^{35}$ The energy differences were found to be very small (mostly below the error bars of total energy calculations) and certainly below the overall uncertainties of our non-additive model.

The pair potential $V_{\text {pair }}^{i}$ between the lead ion and helium atoms has been calculated at the $\operatorname{CCSD}(\mathrm{T})$ level with an extended aug-cc-pV5Z basis and additional bond functions and subsequently represented by the following analytic form with parameters resulting from least squares fitting given in Table 1:

$$
V_{\text {pair }}^{\mathrm{Pb}-\mathrm{He}_{i}}(r)=A e^{-\mathrm{br}}-\sum_{i=4}^{6} \frac{C_{i}}{r^{i}}
$$

The $C_{4}$ coefficient was fixed at 2.76 a.u., consistent with our computed polarizability of $1.38 \mathrm{a}_{0}^{3}$ for helium, in order to guarantee the proper long-range limit (interaction between a charge and an induced dipole) according to eqn (7): The analytical fit together with the asymptotic form is displayed in Fig. 1 and exhibits a well depth of about $810 \mathrm{~cm}^{-1}$ at a $\mathrm{Pb}-\mathrm{He}$ distance of $2.67 \AA$. The presence of other than electrostatic contributions to the binding is clearly visible since the computed curve is more attractive than the charge-dipole interaction. We should emphasize that none of the coefficients beyond $C_{4}$ has a clear physical interpretation and should be regarded as a fitting parameter which ensures a correct interpolation of the ab initio energies.

For the non additive part of our interaction model we need a quantitative description of induced dipole moments on all atoms. Due to the large size of the electron cloud of the lead ion conventional electrostatic formulas for point multipoles are only asymptotically valid. The effective distance dependent polarizability of helium, $\alpha(r)$, was estimated from a SAPT

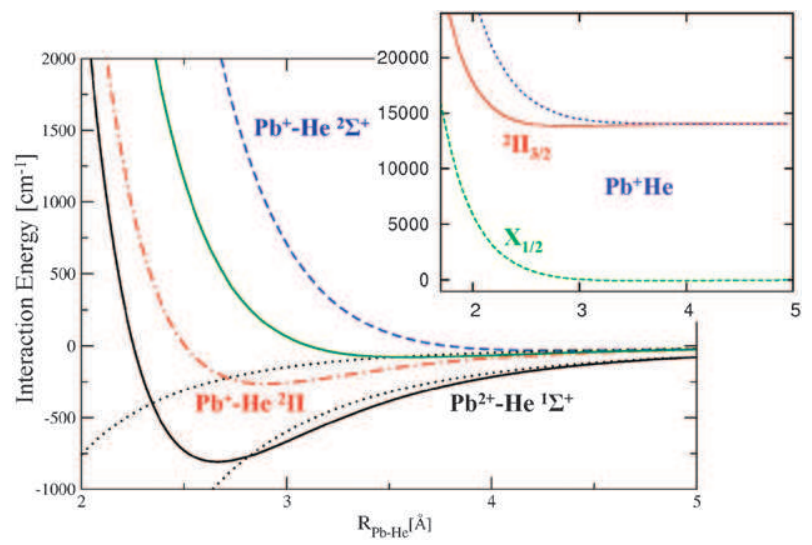

Fig. 1 Ab initio potential curves for the $\mathrm{X}^{1} \Sigma^{+}$state of $\mathrm{Pb}^{2+}-\mathrm{He}$ (solid) and the zero order ${ }^{2} \Pi$ (dash dotted curve) and ${ }^{2} \Sigma^{+}$states (dashed curve) of $\mathrm{Pb}^{+}-\mathrm{He}$ together with the resulting $X$ state (solid curve) arising from spin-orbit mixing. The two dotted curves indicate the expected $q^{2} / R_{\mathrm{Pb}-\mathrm{He}}^{4}, q=1,2$ asymptotes. The inset shows the full set of spin-orbit eigenstates of $\mathrm{Pb}^{+} \mathrm{He}$ and their correlation with the $\mathrm{Pb}^{+}$atomic asymptotes on an expanded energy scale. 
Table 1 Parameters for the analytical representation of pairwise interactions between $\mathrm{Pb}^{2+}$ ions and helium and pairwise interactions between $\mathrm{Pb}^{+}$ions and helium. All parameters are given in atomic units

\begin{tabular}{lccc}
\hline & $\mathrm{Pb}^{2+}-\mathrm{He}$ & $\mathrm{Pb}^{+}-\mathrm{He}$ & \\
\cline { 3 - 4 } & ${ }^{1} \Sigma^{+}$ & ${ }^{2} \Sigma^{+}$ & ${ }^{2} \Pi$ \\
\hline$A$ & 91.3675 & 27.7663 & 70.8869 \\
$B$ & 1.98184 & 1.48046 & 1.96445 \\
$C_{4}$ & 2.76 & 0.69 & 0.69 \\
$C_{5}$ & -10.7639 & -14.0453 & -6.7847 \\
$C_{6}$ & 113.415 & 158.906 & 89.5906 \\
\hline
\end{tabular}

Table 2 Total ground state energies (in $\mathrm{cm}^{-1}$ ) for $\mathrm{Pb}^{2+}-\mathrm{He}_{n}$ and $\mathrm{Pb}^{+}-\mathrm{He}_{n}$ clusters from DMC calculations with several potential models $(\mathrm{SO} /$ Ind $=$ spin-orbit coupling and induction, $\mathrm{SO}=$ only spin-orbit coupling, no $\mathrm{SO}=$ neither spin-orbit coupling nor induction). Numbers in parentheses are $1 \sigma$ statistical uncertainties in units of the last digit

\begin{tabular}{|c|c|c|c|c|}
\hline \multirow[b]{2}{*}{$n$} & \multirow[b]{2}{*}{$\mathrm{Pb}^{2+}-\mathrm{He}_{n}$} & \multicolumn{3}{|l|}{$\underline{\mathrm{Pb}^{+}-\mathrm{He}_{n}}$} \\
\hline & & $\mathrm{SO} / \mathrm{Ind}$ & $\mathrm{SO}$ & No SO \\
\hline 2 & $-1412.9(1)$ & $-113.59(2)$ & & $-422.7(1)$ \\
\hline 3 & $-2091.7(1)$ & $-170.23(2)$ & & $-614.3(1)$ \\
\hline 4 & $-2745.3(1)$ & $-226.69(2)$ & & $-804.5(1)$ \\
\hline 5 & $-3383.5(1)$ & $-282.94(3)$ & & $-987.2(2)$ \\
\hline 6 & $-4006.1(2)$ & $-338.90(3)$ & & $-1131.8(2)$ \\
\hline 7 & $-4596.2(2)$ & $-394.45(3)$ & & $-1167.6(3)$ \\
\hline 8 & $-5164.6(2)$ & $-449.36(4)$ & & $-1203.5(3)$ \\
\hline 9 & $-5700.9(4)$ & $-503.35(5)$ & & $-1240.5(3)$ \\
\hline 10 & $-6186.8(5)$ & $-556.24(4)$ & & $-1276.7(4)$ \\
\hline 11 & $-6608.2(5)$ & $-607.08(6)$ & $-626.1(1)$ & $-1313.2(4)$ \\
\hline 12 & $-7072(1)$ & $-656.76(9)$ & $-679.6(1)$ & $-1347.1(4)$ \\
\hline 13 & $-7295(1)$ & $-701.32(8)$ & $-728.2(2)$ & $-1382.4(4)$ \\
\hline 14 & $-7533(1)$ & $-742.8(1)$ & $-773.4(2)$ & $-1417.4(4)$ \\
\hline 15 & $-7689(2)$ & $-779.8(2)$ & $-814.6(3)$ & $-1447.8(5)$ \\
\hline 16 & $-7764(2)$ & $-811.5(2)$ & $-849.5(4)$ & $-1475.9(6)$ \\
\hline 17 & $-7825(2)$ & $-836.2(2)$ & $-877.9(4)$ & $-1500.6(6)$ \\
\hline 18 & $-7898(3)$ & $-853.1(3)$ & $-897.5(4)$ & $-1523.7(7)$ \\
\hline 19 & & $-864.5(2)$ & $-911.4(4)$ & $-1549.4(7)$ \\
\hline 20 & & $-876.5(3)$ & & $-1567.6(6)$ \\
\hline 21 & & $-884.9(2)$ & & $-1589.7(9)$ \\
\hline 22 & & $-894.2(2)$ & & $-1607.5(10)$ \\
\hline 23 & & $-903.5(3)$ & & $-1627.1(10)$ \\
\hline 24 & & $-913.1(3)$ & & $-1646.1(11)$ \\
\hline 25 & & $-921.6(3)$ & & $-1660.7(11)$ \\
\hline
\end{tabular}

decomposition of the $\mathrm{Pb}^{2+}-\mathrm{He}$ interaction energy and gives induced $\mathrm{He}$ dipole moments according to

$$
\vec{\mu}_{\mathrm{He}}=\frac{q_{\mathrm{Pb}} \alpha(r)}{r^{2}} \vec{e}_{\mathrm{PbHe}}
$$

where $\alpha(r)=1.34541+23.2314 \exp (-0.153667 r)$ and $\vec{e}_{\mathrm{PbHe}}$ is a unit vector pointing from $\mathrm{Pb}$ to $\mathrm{He}$. This induced dipole moment then induces an additional dipole moment (and also higher moments) on the lead atom, which has been calculated as

$$
\vec{\mu}_{\mathrm{Pb}}(r)=\frac{2 \vec{\mu}_{\mathrm{He}} \alpha_{\mathrm{Pb}}}{r^{3}}
$$

with $\alpha_{\mathrm{Pb}}=14.6 \mathrm{a}_{0}^{3}$. After evaluation of the approximate size of higher order contributions we decided to retain in our model only induced dipole moments. The interaction between induced dipole moments $\vec{\mu}_{\mathrm{a}}$ and $\vec{\mu}_{\mathrm{b}}$ separated by a distance $r_{\mathrm{ab}}$ was then calculated using the standard formula

$$
V_{\mu_{\mathrm{a}} \mu_{\mathrm{b}}}=\frac{1}{r_{\mathrm{ab}}^{3}}\left[\vec{\mu}_{\mathrm{a}} \cdot \vec{\mu}_{\mathrm{b}}-3\left(\vec{\mu}_{\mathrm{a}} \cdot \vec{e}_{\mathrm{ab}}\right)\left(\vec{e}_{\mathrm{ab}} \cdot \vec{\mu}_{\mathrm{b}}\right)\right]
$$

We checked in explicit CCSD(T) calculations on $\mathrm{Pb}^{2+} \mathrm{He}_{2}$ that our model captures all essential features of the three body interactions. As a further test we used our model to predict minimum energy structures for larger clusters which we compared with results from DFT optimizations. The energies of the respective minima are displayed in the right panel of Fig. 2 which shows more than satisfactory agreement. In view of the limited validity of DFT calculations for the present system these results should, however, be taken with some caution, but they largely exclude the presence of major defects in our analytical many body model.

$2 \mathbf{P b}^{+}-\mathbf{H e}$. Constructing the potential energy surface for the singly charged $\left(\mathrm{PbHe}_{n}\right)^{+}$clusters is significantly more complicated due to the open shell $\mathrm{s}^{2} \mathrm{p}$ character of the $\mathrm{Pb}^{+}$ ion which leads to a triply degenerate ${ }^{2} \mathrm{P}$ state which splits into ${ }^{2} \Sigma^{+}$and ${ }^{2} \Pi$ states for $\mathrm{Pb}^{+} \mathrm{He}$. Both potential curves were computed at the CCSD(T)/aug-cc-pV5Z (augmented with bond functions) level and fitted to the analytical form given in eqn (9) with the appropriate constraint on the $C_{4}$ coefficient. The resulting potential parameters are given in Table 1 . The fitted potential curves are shown in Fig. 1. Note that also here we observe significant binding contributions beyond electrostatics for the ${ }^{2} \Pi$ state. The minimum of the ${ }^{2} \Pi$ state occurs at $2.9 \AA$ at an interaction energy of $-265 \mathrm{~cm}^{-1}$. The shallow ${ }^{2} \Sigma^{+}$ state has a minimum of $-32.5 \mathrm{~cm}^{-1}$ at an internuclear separation of $4.3 \AA$. The interaction potentials show that the $\mathrm{Pb}^{+}$ion is noticeably larger than $\mathrm{Pb}^{2+}$ and the bonding is significantly weaker. In reality this effect is enhanced by the mixing between the ${ }^{2} \Pi$ and the much more weakly bound ${ }^{2} \Sigma^{+}$state arising from spin-orbit coupling which will be discussed below.

From these pair contributions, we can construct the total potential energy surface within the diatomics-in-molecules framework. ${ }^{43}$ We first define diabatic potentials which depend on the lead-helium distance and the orientation between the $\mathrm{p}$ orbital and the $\mathrm{Pb}-\mathrm{He}$ axis:

$$
U(r, \gamma)=V_{0}(r)+V_{2}(r) P_{2}(\cos \gamma)
$$

where $P_{2}$ is the second Legendre polynomial and $\gamma$ is the angle between the p-orbital orientation and the $\mathrm{Pb}-\mathrm{He}$ vector. The isotropic component $V_{0}(r)$ can be expressed as $\frac{1}{3}\left(V_{\Sigma}+2 V_{\Pi}\right)$ and $V_{2}(r)$ as $\frac{5}{3}\left(V_{\Sigma}-V_{\Pi}\right)$. We then construct the total diabatic energy in a pairwise additive fashion:

$$
U=\sum_{i=1}^{n} \mathrm{He} U_{i}
$$

and we diagonalize this diabatic energy in a basis of the three $\mathrm{p}$ orbitals, retaining the lowest eigenvalue as the total energy. To this eigenvalue we add the induction contribution and the helium-helium interaction.

$$
V=\operatorname{Diag}\left[\left\langle p_{k}|U| p_{l}\right\rangle\right]+\sum_{i=1}^{{ }^{n} \mathrm{He}} \sum_{j=i+1}^{{ }^{n} \mathrm{He}}\left(V_{\text {pair }}^{i j}+V_{\text {ind }}^{i j}\right)
$$



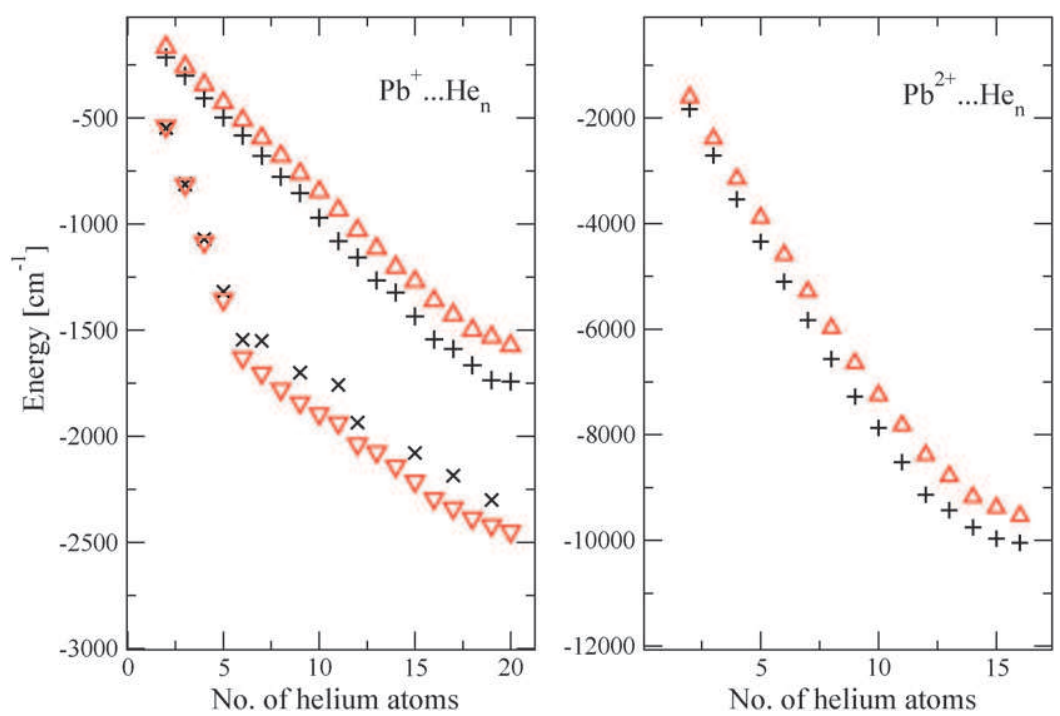

Fig. 2 Energies of global minima for $\mathrm{Pb}^{+} \mathrm{He}_{n}$ (left panel) and $\mathrm{Pb}^{2+} \mathrm{He}_{n}$ (right panel). Note the different energy scales. Triangles indicate energies found in searches on the many body model surfaces and crosses give energies obtained in minimizations using DFT calculations (see text for details). The left panel shows data from calculations with (upper pair of curves) and without (lower pair of curves) inclusion of spin-orbit coupling.

Unlike the $\mathrm{Pb}^{2+}$ case we did not include an induced dipole moment on the lead ion.

Up to this point, the effect of spin-orbit coupling has been neglected. We consider that the spin orbit splitting of the complex is identical to that of the $\mathrm{Pb}^{+}$ion $(\Delta=$ $14081 \mathrm{~cm}^{-144}$ ). The spin-orbit coupling operator $A_{\mathrm{SO}} \hat{L} \hat{S}$ then needs to be included into the diabatization procedure. We therefore have to diagonalize the complex $6 \times 6$ matrix in the spin-orbital basis.

$$
V=\operatorname{Diag}\left[\left\langle p_{k}, \alpha|U| p_{l}, \alpha^{\prime}\right\rangle\right]+\sum_{i=1}^{n^{n} \mathrm{He}} \sum_{j=i+1}^{n_{\mathrm{He}}}\left(V_{\text {pair }}^{i j}+V_{\text {ind }}^{i j}\right)
$$

The spin-orbit splitting $\Delta=3 A_{\mathrm{SO}}$ is so much larger than the zero order separation between the ${ }^{2} \Pi$ and ${ }^{2} \Sigma^{+}$states that this procedure essentially reduces to the weighted isotropic average $V_{0}=\frac{1}{3}\left(V_{\Sigma}+2 V_{\Pi}\right)$ but the full diagonalization procedure was used for the potential evaluation in our DMC calculations.

We tested the quality of our many body model by comparison with explicit DFT calculations on larger clusters with and without inclusion of spin-orbit coupling. The resulting energies are displayed in the left panel of Fig. 2 and show very satisfactory agreement. While performing the calculations without spin-orbit coupling we encountered some difficulties with the convergence of the DFT procedure due to state switching which explains the absence of some data points. The rather different evolution of the total energy with a well defined kink at $n=6$ in the absence of spin-orbit coupling and a smooth behavior for the full calculations is a result of strong electronic anisotropy which disappears due to the strong mixing induced by spin-orbit interaction, an effect on which we will elaborate further below.

\section{B Strong coordination: the case of $\mathbf{P b}^{\mathbf{2}}$}

Shell closure effects and the appearance of so called magic numbers have been reported in theoretical studies of several ion-helium systems ${ }^{16,18,45}$ and are known from experiments. ${ }^{14,15,22,46,47}$ The $\mathrm{Pb}^{2+}-\mathrm{He}$ interaction ranks among the strongest ion-helium interactions known and one might expect the formation of very pronounced snowballs in spite of the small helium mass and resulting large zero point energy. The experimentally observed ion yield distribution ${ }^{47}$ indicates that $n=12$ has special stability. This coordination number also appeared to be special in the theoretical study of Hermann et al. ${ }^{3}$ which found, however, that the first coordination or solvation shell is completed only at $n=15$. In this section we will analyse the necessary distinction between energetic and structural preference.

1 Quantum ground state energetics. The total quantum ground state energies $E_{0}$ relative to separated particles and the incremental energies $E_{0}(n)-E_{0}(n-1)$ from our DMC calculations with and without inclusion of the induction terms are displayed in Fig. 4 and collected in Table 2. Induction terms have a very strong influence on the total ground state energies, in particular for larger clusters. However, the pattern of energy increments is not qualitatively modified by these terms. The evolution of the energy increments can be divided into three regimes: For sizes up to $n=12$ the energy gained through the binding of an additional helium atom is relatively large and smoothly declines until a special stabilization is reached at $n=12$. The sizes $n=13$ to 15 with somewhat unsystematic binding energies form a transition regime to the final regime of weak incremental binding starting at $n=16$. By conventional criteria $n=12$ and $n=14$ would be identified as magic numbers indicating that inclusion of zero point averaging does not lead to a qualitative change of the stabilization energy pattern derived from electronic minima by Hermann $\mathrm{et}_{\mathrm{al}}{ }^{3}$ In the usual fall off of the ion yield with increasing mass, $n=12$ is expected to stick out much more than $n=14$, which is in agreement with the experiment. ${ }^{47}$

2 Structure of $\mathrm{Pb}^{2+} \mathrm{He}_{n}$ clusters. The lowest energy structures encountered in DMC calculations with typically $10^{9}$ 
samples are depicted for selected $\mathrm{Pb}^{2+} \mathrm{He}_{n}$ aggregates in the left column of Fig. 3 and are good estimates for the respective global minimum arrangements. The interaction is dominated by the central field provided by the $\mathrm{Pb}^{2+}$ ion. The resulting classical structures are controlled by the net helium-helium attraction for small $n$ and by the size of the repulsive core of helium atoms for large $n$. At $n=12$ the standard icosahedral symmetry is reached but up to 15 helium atoms can be accommodated at practically identical distances, as previously found. ${ }^{3}$ For $n=16$ one helium atom occupies a more distant position and for $n=18$ one can clearly distinguish 3 atoms in such a situation.

Radial density profiles from DMC calculations for $\mathrm{Pb}^{2+} \mathrm{He}_{n}$ clusters for several relevant sizes $n$ are shown in Fig. 6. The peak helium density increases more or less linearly with $n$ and slowly shifts outward up to $n=12$ where a value of about $0.26 \AA^{-3}$ is reached, corresponding to 12 times the bulk liquid helium density (upper panel of the Figure). By comparison with the density of solid helium $\left(\rho_{\mathrm{He}}=0.214 \mathrm{~kg} \mathrm{l}^{-1}=\right.$ $0.032 \AA^{-3}$ at $1.15 \mathrm{~K}$ and $66 \mathrm{~atm}^{48}$ ) this shell might be called solid already for very small $n$. This interpretation is, however, improper because for most $n$ this shell is structurally disordered. From $n=12$ to $n=15$ a single shell structure is observed which now exhibits a noticeable increase of its width indicative of the progressive disordering of the symmetric packing reached at $n=12$. One could be tempted to interpret

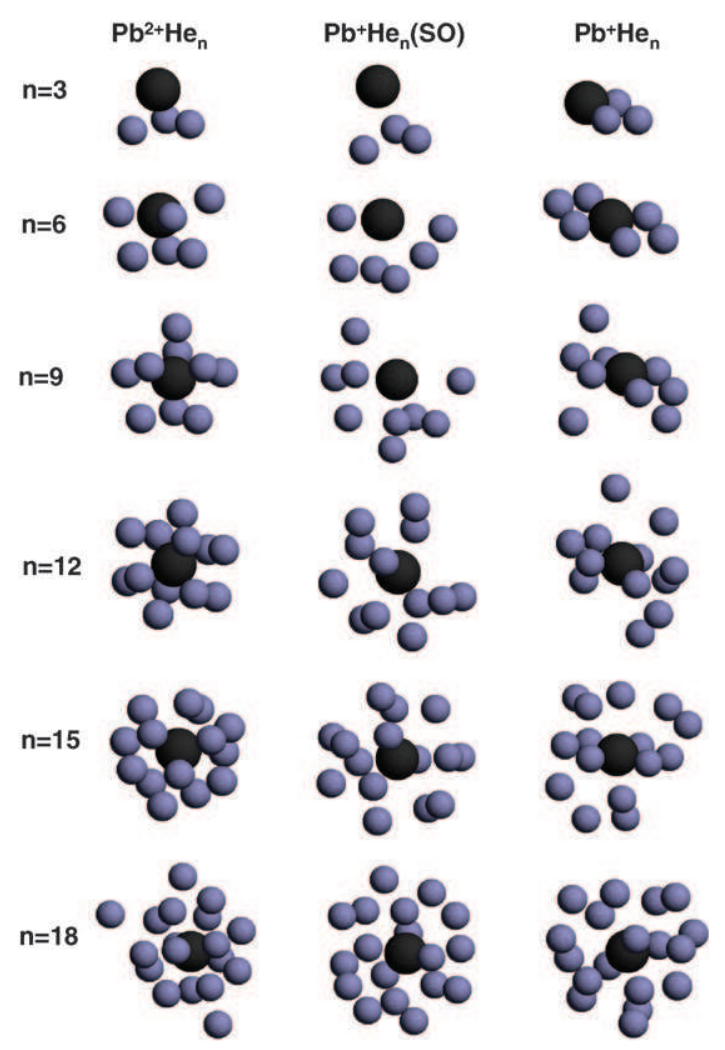

Fig. 3 Lowest energy structures derived from the non-additive model potential for $\mathrm{Pb}^{2+} \mathrm{He}_{n}$ (left column) and $\mathrm{Pb}^{+} \mathrm{He}_{n}$ with (center column) and without (right column) inclusion of spin-orbit coupling. Note the icosahedral structure for $\mathrm{Pb}^{2+} \mathrm{He}_{12}$ and the single shell structure for $\mathrm{Pb}^{2+} \mathrm{He}_{15}$ and pronounced belt (sub)structures for $\mathrm{Pb}^{+} \mathrm{He}_{n}$ if spinorbit coupling is neglected. this transition between an ordered icosahedral structure and disordered structures as some kind of melting or dissolving of the nanocrystal by additional helium. The formation of a second density maximum clearly sets in only at $n=16$. The peak density of this second maximum again exceeds the bulk helium density and in an exploratory calculation for $n=125$ we actually observed a well developed third maximum not shown in the Figure.

Inclusion of zero point delocalization does not destroy the single shell nature of $n=15$, but it is questionable if this system would qualify as a true coordination compound or snowball. The low but appreciable helium density in the minimum between the shells definitely allows particle exchange. The "snowball" of size $n=15$ will probably not keep its identity and should be viewed as a dynamical object involved in exchange with its environment.

\section{Weak coordination: the case of $\mathrm{Pb}^{+}$}

The $\mathrm{Pb}^{+}$ion is noticeably larger than the $\mathrm{Pb}^{2+}$ ion and could therefore allow larger coordination numbers. The much weaker interaction with helium would tend, however, to render the structures less rigid, which can make structural transitions less obvious. The structure of the $\mathrm{Pb}^{+} \mathrm{He}_{n}$ aggregates is profoundly affected by the strong spin-orbit interaction which almost eliminates the anisotropy. The mass spectra recorded by Döppner et al. ${ }^{47}$ show a drop of the ion count rate after $n=17$ which could be taken as andication of shell closure.

1 Quantum ground state energetics. The total ground state energies given in Table 2 and depicted in the left panel of Fig. 5 nicely illustrate the importance of spin-orbit coupling. The lower curve gives energies computed without accounting for this effect and indicates systematically lower energies (more binding) than the upper series of triangles which show ground state energies for the complete model with induction and spin-orbit coupling. The lower energies result from essentially $\Pi$-type interactions and the pronounced kink at $n=6$ is due to the strong anisotropy of this model. The essentially isotropic spin-orbit model leads to ground state energies with a rather smooth evolution with cluster size and a slow transition to a weak binding regime which sets in at $n=18$. These trends are more clearly visible in the right panel of Fig. 5 which displays the energy gained upon addition of a helium atom. In comparison with the experimental evidence $n=17$ seems to be the last member of the more strongly binding regime. The effect is, however, rather weak and $n=17$ does not qualify as a magic number.

2 Structure of $\mathbf{P b}^{+} \mathbf{H e}_{n}$ clusters. The two rightmost columns of Fig. 3 show lowest energy particle arrangements visited during very long DMC runs and are representative of the global minima as already discussed in our comparison between potential model minima and explicit DFT calculations. The central column showing the structural evolution for the full and almost isotropic model indicates the initial formation of a polar cap and progressive coating of the central atom eventually limited by the repulsive helium cores. This is similar 

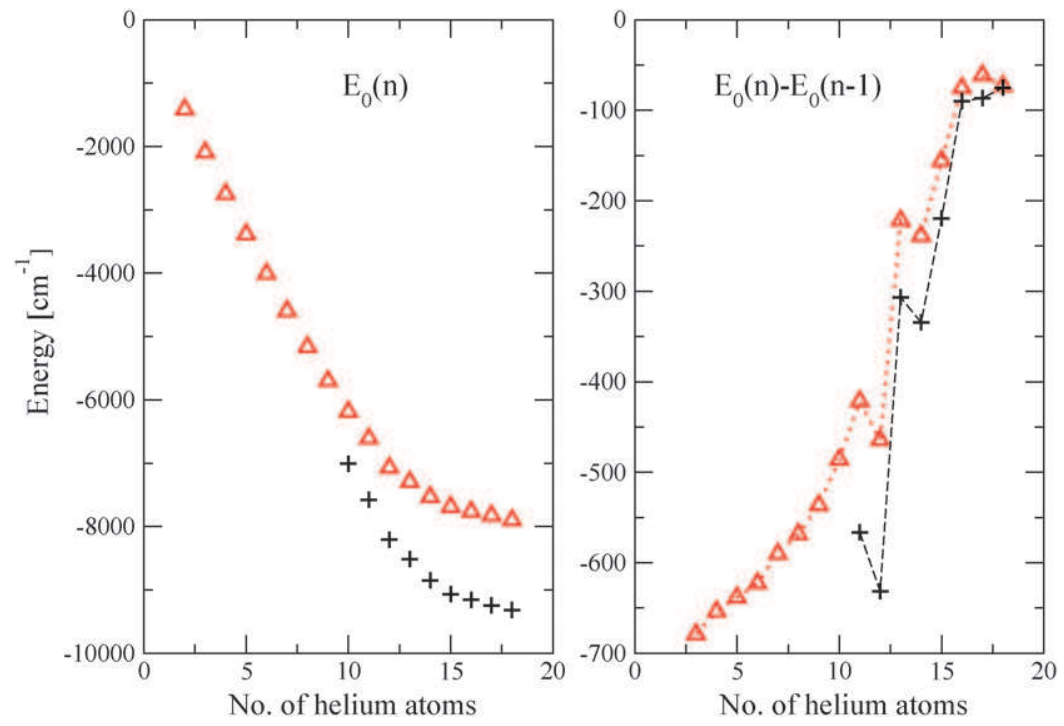

Fig. 4 Total ground state energies (left panel) and energy increments (right panel) for $\mathrm{Pb}^{2+} \mathrm{He}_{n}$ from DMC calculations using the full interaction model with induction terms (triangles). Crosses indicate results obtained upon neglect of induction for selected sizes $n$. Note the consistent appearance of 'magic' sizes at $n=12$ and $n=14$ in both models in spite of the significant contribution of induction to the total energies.

to $\mathrm{Pb}^{2+}$ but with the important difference of looser structures. The largest single shell system is $n=17$.

The physically unrealistic model without spin-orbit coupling is, however, interesting since it resembles other cases of atoms in P-states interacting with rare gas atoms. ${ }^{49,50}$ Like in these other systems we observe preferential filling of a very compact belt in the nodal plane of the p-orbital followed by occupation of much less favored out of plane positions. The helium atoms try to avoid the region of maximum electron density described by the p-orbital. This is exactly the mechanism which leads to the formation of "bubbles" in liquid and solid helium around certain dopants.

Selected radial density profiles for $\mathrm{Pb}^{+} \mathrm{He}_{n}$ clusters are displayed in Fig. 7. The first shell of helium atoms aggregates around $\mathrm{Pb}^{+}$with a continuous and almost linear build up of density until a maximum of about $0.09 \AA^{-3}$ is reached at $n=17$, a little more than four times the bulk liquid helium density. This size appears to represent closure of some sort of shell consistent with our classical structures. This peak density value is again far above the density of solid helium, but remains significantly below the values calculated for $\mathrm{Pb}^{2+}$. The onset of the build up of a second shell is much less pronounced than for $\mathrm{Pb}^{2+}$ and initially one only sees a broadening of the first peak and a prolongation of its tail. Only at $n=25$ a clearly identifiable density minimum between first and second neighbor peaks is observed. The density at its minimum is near half the bulk liquid helium density and well above the values found for $\mathrm{Pb}^{2+}$ again allowing efficient
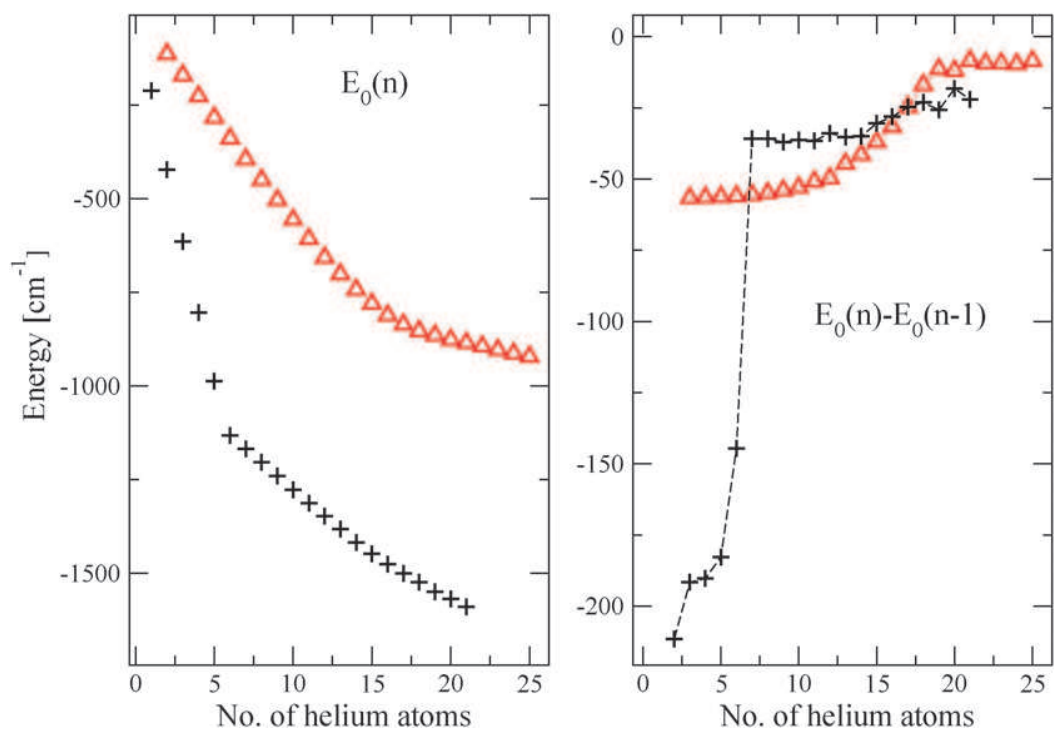

Fig. 5 Total ground state energies (left panel) and energy increments (right panel) for $\mathrm{Pb}^{+} \mathrm{He}_{n}$ from DMC calculations using the full interaction model with induction terms and spin-orbit coupling (triangles) and a model without induction and spin-orbit coupling (crosses). Note the pronounced signature of a belt filling structure at $n=6$ without spin-orbit coupling and the smooth evolution upon inclusion of this effect. 

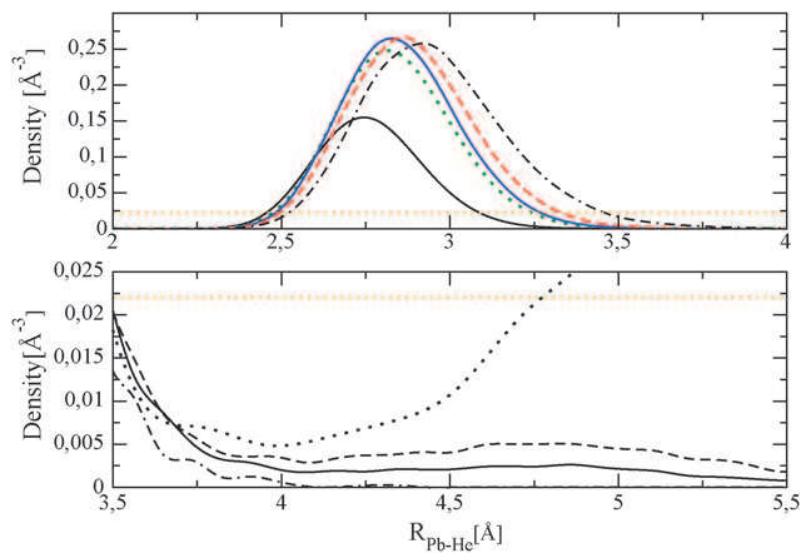

Fig. 6 Radial helium densities for selected $\mathrm{Pb}^{2+} \mathrm{He}_{n}$ clusters from DMC calculations. The upper panel shows the evolution of the nearest neighbor density peak for $n=6$ (dark solid), $n=11$ (dotted), $n=12$ (light solid), $n=13$ (dashed) and $n=15$ (dash dotted) with clear density saturation at $n=12$. The lower panel shows the onset and evolution of the second density maximum for $n=15$ (dash dotted), $n=16$ (solid), $n=17$ (dashed) and $n=125$ (dotted). Note the presence of a significant helium density in the minimum and the difference in the density scale of the two panels. The horizontal dotted lines indicate the bulk density of pure helium $\left(0.02197 \AA^{-3}\right.$ at $2.17 \mathrm{~K}$ and saturation pressure).

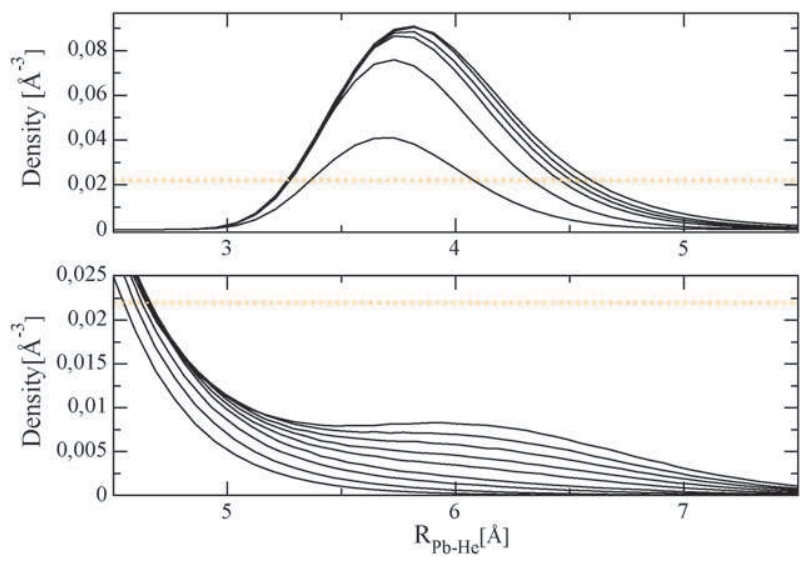

Fig. 7 Radial helium densities for selected $\mathrm{Pb}^{+} \mathrm{He}_{n}$ clusters. The upper panel shows the evolution of the nearest neighbor density peak for $n=6, n=12, n=15, n=16, n=17$ and $n=18$ with saturation clearly reached at $n=17$. The lower panel shows the onset and evolution of the second density maximum for $n=17-25$. The horizontal dotted lines indicate the bulk density of pure helium. Note the difference in the density scale of the two panels.

exchange of helium atoms. Even though for this system the kissing number is very high (at least 17) the shells are not clearly distinguishable.

\section{Conclusion}

We have presented the construction of reliable and very efficient many body models for $\mathrm{Pb}^{2+}$ and $\mathrm{Pb}^{+}$ions interacting with helium atoms based on high level ab initio calculations. These models have been successfully tested against DFT calculations on large clusters. Several similar interesting open shell systems could be treated with the methodology developed in the present study. The inclusion of spin-orbit effects qualitatively changes the structures of $\mathrm{Pb}^{+} \mathrm{He}_{n}$.

The energy scales for $\mathrm{Pb}^{2+} \mathrm{He}_{n}$ and $\mathrm{Pb}^{+} \mathrm{He}_{n}$ were found to be very different and to be reflected in the resulting quantum ground state properties studied by diffusion quantum Monte Carlo. We have studied the influence of quantum delocalization on the structure and energetics of these systems. The computed stability patterns agree well with the available experimental data. In particular we reproduce the magic number of $n=12$ for $\mathrm{Pb}^{2+} \mathrm{He}_{n}$ and observe a transition between two binding regimes near $n=17$ for $\mathrm{Pb}^{+} \mathrm{He}_{n}$.

Both systems exhibit high coordination with respect to classical minimum energy structures (shell closure at $n=15$ for $\mathrm{Pb}^{2+} \mathrm{He}_{n}$ and at $n=17$ for $\left.\mathrm{Pb}^{+} \mathrm{He}_{n}\right)$. Analysis of the quantum distributions shows, however, that the first coordination shell is essentially complete already at $n=12$ for $\mathrm{Pb}^{2+} \mathrm{He}_{n}$ (maximum nearest neighbor density is reached). The addition of further helium atoms partially degrades this compact structure without, however, initiating the formation of a new shell before $n=16$. Once enough atoms are available a distinct second shell starts to build up. Both $n=12$ and $n=15$ are special sizes in the sense that they are the last members of a structural or energetic motive.

The first solvation shell for $\mathrm{Pb}^{+}$is completed at $n=17$ as indicated by the density maximum. Energetically this system does not possess special stability, but it is the last member of an initial binding motive and therefore plays a role comparable to $n=15$ in $\mathrm{Pb}^{2+} \mathrm{He}_{n}$. The second shell is, however, much less clearly distinguished from the first shell.

Even though both systems allow a very large number of nearest neighbors they do not force us to revise the conventional notion of coordination numbers because the limiting sizes (15 and 17, respectively) are highly disordered and the atoms in this first shell cannot be clearly separated from the 'solvent' atoms.

\section{Acknowledgements}

ML would like to acknowledge many fruitful discussions with M. Mladenović and generous financial support by Agence Nationale de la Recherche through grant ANR-08-BLAN0146-01. Access to the high performance computers at IDRIS, Orsay, through grant 82170 as well as computational support of the Center for complex molecular systems and biomolecules is gratefully acknowledged. PS acknowledges support by the Ministry of Education of the Czech Republic (research project No. 6046137307).

\section{References}

1 A. Wouterse, M. Plapp and A. P. Philipse, J. Chem. Phys., 2005, 123, 054507.

2 J. Ribas Gispert, Coordination Chemistry, Wiley VCH, Weinheim, 2008.

3 A. Hermann, M. Lein and P. Schwerdtfeger, Angew. Chem., Int. Ed., 2007, 46, 2444.

4 R. Wesendrup, M. Pernpointner and P. Schwerdtfeger, Phys. Rev. A: At., Mol., Opt. Phys., 1999, 60, R3347.

5 R. Wesendrup, G. E. Moyano, M. Pernpointner and P. Schwerdtfeger, J. Chem. Phys., 2002, 117, 7506. 
6 J. P. Toennies and A. F. Vilesov, Annu. Rev. Phys. Chem., 1998, 49, 1

7 J. P. Toennies and A. F. Vilesov, Angew. Chem., Int. Ed., 2004, 43, 2622.

8 F. Stienkemeier and K. K. Lehmann, J. Phys. B: At., Mol. Opt. Phys., 2006, 39, R127.

9 M. Barranco, R. Guardiola, S. Hernandez, R. Mayol, J. Navarro and M. Pi, J. Low Temp. Phys., 2006, 142, 1.

10 J. Tiggesbäumker and F. Stienkemeier, Phys. Chem. Chem. Phys., 2007, 9, 4748 .

11 W. Koch, G. Frenking, J. Gauss, D. Cremer and J. R. Collins, J. Am. Chem. Soc., 1987, 109, 5917.

12 F. Grandinetti, Int. J. Mass. Spec. Ion. Proc., 2004, 237, 243.

13 K. R. Atkins, Phys. Rev., 1959, 116, 1339.

14 T. Döppner, T. Diederich, A. Przystawik, N. X. Truong, T. Fennel, J. Tiggesbäumker and K.-H. Meiwes-Broer, Phys. Chem. Chem. Phys., 2007, 9, 4639.

15 F. F. da Silva, P. Waldburger, S. Jaksch, A. Mauracher, S. Denifl, O. Echt, T. D. Märk and P. Scheier, Chem.-Eur. J., 2009, 15, 7101.

16 E. Bodo, E. Coccia, D. Lopez-Durán and F. A. Gianturco, Phys. Scr., 2007, 76, C104.

17 E. Coccia, E. Bodo, F. Marinetti, F. A. Gianturco, E. Yildrim, M. Yurtsever and E. Yurtsever, J. Chem. Phys., 2007, 126, 124319

18 S. Paolini, F. Ancilotto and F. Toigo, J. Chem. Phys., 2007, 126, 124317.

19 E. Coccia, E. Bodo and F. A. Gianturco, Europhys. Lett., 2008, 82, 23001.

20 P. Slavíček, M. Roeselová, P. Jungwirth and B. Schmidt, J. Chem. Phys., 2001, 114, 1539.

21 P. Slavíček, P. Jungwirth, M. Lewerenz, N. H. Nahler, M. Fárník and U. Buck, J. Phys. Chem. A, 2003, 107, 7743.

22 C. A. Brindle, M. R. Prado, K. C. Janda, N. Halberstadt and M. Lewerenz, J. Chem. Phys., 2005, 123, 064312.

23 S. F. Boys and F. Bernardi, Mol. Phys., 1970, 19, 553.

24 B. Metz, H. Stoll and M. Dolg, J. Chem. Phys., 2000, 113, 2563.

25 D. E. Woon and T. H. Dunning, Jr, J. Chem. Phys., 1994, 100, 2975.

26 C. Adamo and V. Barone, J. Chem. Phys., 1999, 110, 6158.

27 B. Jeziorski, R. Moszynski and K. Szalewicz, Chem. Rev., 1994, 94, 1887.

28 MOLPRO is a package of $a b$ initio programs written by H.-J. Werner, P. J. Knowles, R. Lindh and F. R. Manby others, see http://www.molpro.net, 2006.
29 D. E. Bernholdt, E. Apra, H. A. Fruchtl, M. F. Guest, R. J. Harrison, R. A. Kendall, R. A. Kuteh, X. Long, J. B. Nicholas, J. A. Nichols, H. L. Taylor and A. T. Wong, Int. J. Quantum Chem., 1995, 29, 475.

30 J. B. Anderson, J. Chem. Phys., 1975, 63, 1499.

31 P. J. Reynolds, D. M. Ceperley, B. J. Alder and W. A. Lester, Jr, J. Chem. Phys., 1982, 77, 5593.

32 C. J. Umrigar, M. P. Nightingale and K. J. Runge, J. Chem. Phys., 1993, 99, 2865.

33 B. L. Hammond, W. A. Lester, Jr and P. J. Reynolds, Monte Carlo Methods in ab initio Quantum Chemistry, World Scientific, Singapore, 1994.

34 D. Blume, M. Lewerenz, F. Huisken and M. Kaloudis, J. Chem. Phys., 1996, 105, 8666

35 M. Lewerenz, J. Chem. Phys., 1997, 106, 4596.

36 S. A. Chin, Phys. Rev. A: At., Mol., Opt. Phys., 1990, 42, 6991.

37 P. Håkansson, M. Mella, D. Bressanini, G. Morosi and M. Patrone, J. Chem. Phys., 2006, 125, 184106.

38 M. H. Kalos, J. Comput. Phys., 1967, 2, 257.

39 R. N. Barnett, P. J. Reynolds and W. A. Lester, Jr, J. Comput. Phys., 1991, 96, 258.

40 W. H. Press, S. A. Teukolsky, W. T. Vetterling and B. P. Flannery, Numerical Recipes in FORTRAN. The Art of Scientific Computing, Cambridge University Press, Cambridge, 2nd edn, 1992.

41 R. A. Aziz, F. R. McCourt and C. C. K. Wong, Mol. Phys., 1987, 61, 1487.

42 M. Jeziorska, W. Cencek, K. Patkowski, B. Jeziorski and K. Szalewicz, J. Chem. Phys., 2007, 127, 124303.

43 A. I. Krylov, R. B. Gerber and R. D. Coalson, J. Chem. Phys., 1996, 105, 4626

44 C. E. Moore, Atomic Energy Levels, NBS, Washington, 1952.

45 F. Marinetti, E. Bodo and F. A. Gianturco, ChemPhysChem, 2007, 8, 93.

46 T. M. Kojima, N. Kobayashi and Y. Kaneko, Z. Phys. D: At., Mol. Clusters, 1992, 22, 645.

47 T. Döppner, T. Diederich, S. Göde, A. Przystawik, J. Tiggesbäumker and K.-H. Meiwes-Broer, J. Chem. Phys., 2007, 126, 244513.

48 D. G. Henshaw, Phys. Rev., 1958, 109, 328.

49 M. Leino, A. Viel and R. E. Zillich, J. Chem. Phys., 2008, 129, 184308.

50 P. Moroshkin, A. Hofer and A. Weis, Phys. Rep., 2008, 469, 1. 